\title{
Towards Syntactic Iberian Polarity Classification *
}

\author{
David Vilares $^{\star \dagger}$, Marcos Garcia ${ }^{\star \ddagger}$, Miguel A. Alonso ${ }^{\uparrow \dagger}$, Carlos Gómez-Rodríguez ${ }^{\natural \uparrow \dagger}$ \\ Universidade da Coruña \\ *FASTPARSE Lab, "LyS Group \\ † Departamento de Computación, Campus de Elviña \\ ${ }^{\ddagger}$ Departamento de Letras, Campus da Zapateira \\ 15701, A Coruña, Spain \\ david.vilares@udc.es, marcos.garcia.gonzalez@udc.es \\ miguel.alonsodudc.es, carlos.gomez@udc.es
}

\begin{abstract}
Lexicon-based methods using syntactic rules for polarity classification rely on parsers that are dependent on the language and on treebank guidelines. Thus, rules are also dependent and require adaptation, especially in multilingual scenarios. We tackle this challenge in the context of the Iberian Peninsula, releasing the first symbolic syntax-based Iberian system with rules shared across five official languages: Basque, Catalan, Galician, Portuguese and Spanish. The model is made available. ${ }^{1}$
\end{abstract}

\section{Introduction}

Finding the scope of linguistic phenomena in natural language processing (NLP) is a core utility of parsing. In sentiment analysis (SA), it is used to address structures that play a role in polarity classification, both in supervised (Socher et al., 2013) and symbolic (Vilares et al.) models. In the latter case, these are mostly monolingual and dependent on the annotation of the training treebank, and so the rules are annotation-dependent too. Advances in NLP make it now possible to overcome such issues. We present a model that analyzes five official languages in the Iberian Peninsula: Basque $(e u)$, Catalan ( $c a)$, Galician $(g l)$, Portuguese ( $p t)$ and Spanish (es). We rely on three premises:

1. Syntactic structures can be defined in a universal way (Nivre et al., 2015).

* DV was funded by MECD (FPU13/01180). MG is funded by a Juan de la Cierva grant (FJCI-2014-22853). CGR has received funding from the ERC, under the European Union's Horizon 2020 research and innovation programme (FASTPARSE, grant agreement No 714150). This research was supported by MINECO (FFI2014-51978-C2).

${ }^{1}$ The resources used in this work have been integrated as a part of https://github.com/aghie/uuusa
2. Training a single model for multilingual parsing is feasible (Ammar et al., 2016).

3 . We can define universal rules for various phenomena, if 1 is assured (Vilares et al., 2017).

Based on those, we: (a) combine existing subjectivity lexica, (b) train an Iberian tagger and parser, and (c) define a set of Iberian syntax-based rules. The main contributions of the paper are:

1. A single set of syntactic rules to handle linguistic phenomena across five Iberian languages from different families.

2. The first end-to-end multilingual syntax-based SA system that analyzes five official languages of the Iberian Peninsula. This is also the first evaluation for SA that provides results for some of them.

\section{Related work}

Polarity classification has been addressed through machine learning (Mohammad et al., 2013; Socher et al., 2013; Vo and Zhang, 2016), and lexiconbased models (Turney, 2002). Most of the research involves English texts, although studies can be found for other languages such as Chinese (Chen and Chen, 2016) or Arabic (Shoukry and Rafea, 2012).

For the official languages in the Iberian Peninsula, much of the literature has focused on Spanish. Brooke et al. (2009) proposed a lexiconbased SA system that defines rules at the lexical level to handle negation, intensification or adversative subordinate clauses. They followed a crosslingual approach, adapting their English method (Taboada et al., 2011) to obtain the semantic orientation (SO) of Spanish texts. Vilares et al. created a syntactic rule-based system, by making an interpretation of Brooke et al.'s system, but limited to AnCora trees (Taulé et al., 2008). MartínezCámara et al. (2011) were one of the first to report a wide set of experiments on a number of 
bag-of-words supervised classifiers. The TASS workshop on sentiment analysis focused on Spanish language (Villena-Román et al., 2013) annually proposes different challenges related to polarity classification, and a number of approaches have used its framework to build their Spanish systems, most of them based on supervised learning (Saralegi and San Vicente, 2013; Gamallo et al., 2013; Hurtado et al., 2015; Vilares et al., 2015).

Sentiment analysis for Portuguese has also attracted the interest of the research community. Silva et al. (2009) presented a system for detection of opinions about Portuguese politicians. Souza et al. (2011) built a lexicon for Brazilian Portuguese exploring different techniques (e.g. translation and thesaurus-based approaches) and available resources. Souza and Vieira (2012) carried out a study of Twitter data, exploring preprocessing techniques, subjectivity data and negation approaches. They concluded that those have a small impact on the polarity classification of tweets. Balage Filho et al. (2013) evaluate the quality of the Brazilian LIWC dictionary (Pennebaker et al., 2001) for SA, comparing it with existing lexica for this language.

For Basque, Catalan and Galician, literature is scarce. Cruz et al. (2014) introduce a method to create multiple layered lexicons for different languages including co-official languages in Spain. San Vicente and Saralegi (2016) explore different ways to create lexicons, and apply them to the Basque case. They report an evaluation on a Basque dataset intended for polarity classification. Bosco et al. (2016) discuss the collection of data for the Catalan Elections and design an annotation scheme to apply SA techniques, but the dataset is still not available. With respect to Galician, in this article we will present the first published results for this language.

\section{SISA: Syntactic Iberian SA}

\subsection{Preliminaries}

Vilares et al. (2017) propose a formalism to define compositional operations. Given a dependency tree for a text, a compositional operation defines how a node in the tree modifies the semantic orientation (SO) of a branch or node, based on elements such as the word form, part-of-speech (PoS) tag or dependency type, without limitations in terms of its location inside such tree. They released an implementation, where an ar-

\begin{tabular}{|c|rrrrr|}
\hline Tag & es & pt & ca & eu & gl \\
\hline \hline ADJ & 2,045 & 1,865 & 1,686 & 1,757 & 2,002 \\
NOUN & 1,323 & 1,183 & 1,168 & 1,211 & 1,270 \\
ADV & 594 & 570 & 533 & 535 & 599 \\
VERB & 739 & 688 & 689 & 563 & 723 \\
\hline
\end{tabular}

Table 1: Size of the SFU (single words) lexica.

bitrary number of practical compositional operations can be defined. The system queues and propagates them through the tree, until the moment they must be dequeued and applied to their target. The authors showed how the same set of operations, defined to work under the Universal Treebank (UT) guidelines (McDonald et al., 2013), can be shared across languages, but they do not explore how to create a single pipeline for analyzing many languages. This paper explores that path in the context of Iberian Peninsula, presenting an unified syntactic Iberian SA model (SISA).

We below present how to build SISA, from the bottom (subjectivity lexica, tagging and dependency parsing) to the top levels (application of compositional operations to compute the final SO).

\subsection{Subjectivity Lexica}

SIS A needs multilingual polarity lexica in order to predict the sentiment of a text. We used two sets of monolingual lexica as our starting points:

1. Spanish SFU lexicon (Brooke et al., 2009): It contains SO's for subjective words that range from 1 to 5 for positive and negative terms. We translated it to $c a, e u, g l$ and $p t$ using apertium (Forcada et al., 2011). We removed the unknown words and obtained the numbers in Table $1 .^{2}$

2. ML-Senticon (Cruz et al., 2014): Multi-layered lexica (not available for $p t$ ) with so's where each layer contains a larger number of terms, but less trustable. We used the seventh layer for each language. As $e u, c a$ and $g l$ files have the same PoStag for adverbs and adjectives, they were automatically classified using monolingual tools (Agerri et al., 2014; Padró and Stanilovsky, 2012; Garcia and Gamallo, 2015) (Table 2 contains the statistics). SO's (originally from 0 to 1 ) were linearly transformed to the scale of the SFU lexicon.

The SFU and ML-Senticon lexica for each language were combined to obtain larger monolingual resources, and these were in turn combined

\footnotetext{
${ }^{2}$ We used the original apertium outputs, except for the $p t$ and $g l$ lexica (manually reviewed by a linguist).
} 


\begin{tabular}{|c|rrrr|}
\hline Tag & es & ca & eu & gl \\
\hline \hline ADJ & 2,558 & 1,619 & 22 & 1,530 \\
NOUN & 2,094 & 1,535 & 1,365 & 579 \\
ADV & 117 & 23 & 3 & 26 \\
VERB & 603 & 500 & 272 & 144 \\
\hline
\end{tabular}

Table 2: Size of the resulting ML-Senticon lexica.

\begin{tabular}{|l|rrrrrr|}
\hline Tag & es & pt & ca & eu & gl & Iberian \\
\hline \hline ADJ & 3,775 & 1,865 & 2,704 & 1,529 & 2,990 & 9,385 \\
NOUN & 3,079 & 1,183 & 2,377 & 2,392 & 1,684 & 8,733 \\
ADV & 665 & 570 & 545 & 485 & 612 & 1,891 \\
VERB & 1,177 & 688 & 1,034 & 728 & 801 & 2,998 \\
\hline
\end{tabular}

Table 3: Size of the final lexica.

into a common Iberian lexicon (see Table 3). When merging lexica, we must consider that:

1. In monolingual mergings, the same word can have different SO's. E.g., the Catalan adjective 'abandonat' (abandoned) has -1.875 and -3 in ML-Senticon and SFU, respectively.

2. When combining lexica of different languages, the same word form might have different meanings (and SOs) in each language. Merging them in a multilingual resource could be problematic. For example, the adjective 'espantoso' has a value of -4.1075 in the combined es lexicon (frightening), and of -3.125 in the $g l$ one (frightening), while the same word in the $p t$ data (astonishing) has a positive value of 5 . Note, however, that even if they could be considered very similar from a lexical or morphological perspective, many phonological false friends have different spellings in each language (such as the negative 'vessar' (to spill) in $c a$ and the positive 'besar' (to kiss) in es), so these cases end up not being a frequent problem (only $0.36 \%$ of the words have both positive and negative polarity in the monolingual lexica).

These two problems were tackled by averaging the polarities of words with the same form. Thus, the first monolingual mergings produced a balanced SO (e.g., 'abandonat' has -2.4375 in the combined $c a$ lexicon), while in the subsequent multilingual fusion, contradictory false friends have a final value close to no polarity (e.g., 'espantoso', with a SO of -0.7 in the Iberian lexicon). The impact of these mergings is analyzed in $\S 4$.

\subsection{PoS-tagging and dependency parsing}

For the compositional operations to be triggered, we first need to do the tagging and the dependency parse for a sentence. To do so, we trained an
Iberian PoS-tagger and parser, i.e. single modules that can analyze Iberian languages without applying any language identification tool. Multilingual taggers and parsers can be trained following approaches based on (Vilares et al., 2016; Ammar et al., 2016). We are relying on the Universal Dependency (UD) guidelines (Nivre et al., 2015) to train these tools, since they provide corpora for all languages studied in this paper.

For the Iberian tagger we relied on Toutanova and Manning (2000), obtaining the following accuracies (\%) in the monolingual UD test sets: $p t$ (95.96), es (94.37), ca (97.41), eu (93.88) and $g l$ (94.09). For the Iberian parser we used the approach by Vilares et al. (2016), whose performance (LAS/UAS) ${ }^{3}$ on the same UD test sets was: $p t$ (78.78/84.50), es (80.20/85.23), cat (84.01/88.08), $e u(62.01 / 71.64)^{4}$ and $g l(75.65 / 82.11)$.

\subsection{Compositional operations}

For a detailed explanation of compositional operations, we encourage the reader to consult Vilares et al. (2017), but we here include an overview as part of SISA. Briefly, a compositional operation is tuple $o=(\tau, C, \delta, \pi, S)$ such that:

- $\tau: \mathbb{R} \rightarrow \mathbb{R}$ is a transformation function to apply on the semantic orientation of nodes, where $\tau$ can be weighting $_{\beta}(S O)=S O \times(1+\beta)$ or $\operatorname{shift}_{\alpha}(S O)=\left\{\begin{array}{ll}S O-\alpha & \text { if } S O \geqq 0 \\ S O+\alpha & \text { if } S O<0\end{array}\right.$,

- $C: V \rightarrow\{$ true, false $\}$ is a predicate that determines whether a node in the tree will trigger the operation, based on word forms, PoS-tags and dependency types,

- $\delta \in \mathbb{N}$ is a number of levels that we need to ascend in the tree to calculate the scope of $o$, i.e., the nodes of $T$ whose $\mathrm{SO}$ is affected by the transformation function $\tau$,

- $\pi$ is a priority used to break ties when several operations coincide on a given node, and

- $S$ is a scope function that will be used to determine the nodes affected by the operation.

${ }^{3}$ LAS/UAS: The percentage of arcs where both the head and dependency type / the head are correct.

${ }^{4}$ The parsing results for Basque (with a high proportion of non-projective trees) were worse than expected. However, the parser trained based on the method by Vilares et al. (2016) automatically selected a projective algorithm for training, as the average prevalence of non-projectivity across our five Iberian languages is low. We hypothesize that this is the main reason of the lower performance for this language. 
We adapt the UT operations used by Vilares et al. (2017) to the UD style to handle, which are now described:

1. Intensification: It diminishes or amplifies the so of a word or a phrase. It operates from adjectives or adverbs modifying the so of the head structure they depend on: e.g., the so of 'grande' (big, in es) increases from 1.87 to 2.34 if a word such as 'muy' (very) depends on it and its labeled with the dependency type advmod. Formally, for $\mathrm{o}_{\text {intensification, }}$, $\tau=$ weight $_{\beta}(S O), C=w \in$ intensifiers $\wedge$ $t \in\{$ ADV,ADJ $\} \wedge d \in\{$ advmod,amod,nmod $\}, \delta=1$, $\pi=3$ and $S=\{$ target node, b(advmod), $b($ amod $)\}$, where $b(x)$ indicates that the scope is the first branch at the target level whose dependency type is $x$. $\beta$ is extracted from a lexicon with booster values (in this work obtained from SFU, where 'muy' has a booster value of 0.25 ).

2. Subordinate adversative clauses: This rule is designed for dealing with structures coordinated by adversative conjunctions (such as but), which usually involve opposite polarities between the two joint elements (e.g., "good but expensive"). Here, the so of the first element is multiplied by $1-0.25$, so its polarity decreases. Formally, $\tau=$ weight $_{-0.25}(S O), C=w \in$ adversatives $\wedge$ $t \in\{\mathrm{CONJ}, \mathrm{SCONJ}\} \wedge d \in\{\mathrm{cc}$, advmod,mark $\}, \delta=1$, $\pi=1$ and $S=\{$ subjl $\}$. Subjl indicates that the scope is the first left branch with SO $!=0$ at the target level.

3. Negation: In most cases, negative adverbs shift the polarity of the structures they depend on ("It is nice" versus "It is not nice"). In order to handle these cases, the present rule shifts the polarity of the head structures of a negative adverb by $\alpha$ (where $\alpha=4$, in our experiments). In the previous example, the polarity of "nice" would drop from 3.5 to -0.5 if affected by the rule. Formally, for $\mathrm{o}_{\text {negation }}, \tau=\operatorname{shift}_{4}(S O), C=w \in$ negators $\wedge$ $d \in\{$ neg,advmod $\}, \delta=1, \pi=2$ and $S=\{$ target node, $b$ (root), b(cop), b(nsubj), subjr, all $\}$. Subjr indicates that the scope is the first branch with SO $!=0$ and all indicates to apply negation at the target level as a backoff option, if none of the previous scopes matched.

4. 'If' irrealis: In conditional statements, a SA system may obtain an incorrect polarity due to the presence of polarity words which actually do not reflect a real situation ("This is good" vs "If this is good"). This rule attempts to better analyze these structures by shifting the polarity (here, multiplied by -1 ) if a conditional conjunction depends on it. Formally, for $\mathrm{o}_{\text {irrealis }}, \tau=$ weight $_{-1}(S O)$, $C=w \in$ irrealis $\wedge d \in$ \{mark,advmod,cc $\}, \delta=1$, $\pi=3$ and $S=\{$ target node, subjr $\}$.

\section{Evaluation}

This section presents the results of the experiments we carried out with our system using both the monolingual and the multilingual lexica, compared to the performance of a supervised classifier for three of the five analyzed languages.

\subsection{Testing corpora}

- Spanish SFU (Brooke et al., 2009): A set of 400 long reviews (200 positive, 200 negative) from different domains such as movies, music, computers or washing machines.

- Portuguese SentiCorpus-PT 0.1 (Carvalho et al., 2011): A collection of comments from the Portuguese newspaper Público with polarity annotation at the entity level. As our system assigns the polarity at the sentence level, we selected the SentiCorpus sentences with (a) only one SO and (b) with $>1$ so iff all of them were the same, generating a corpus with 2,086 (from 2,604 ) sentences.

- Basque Opinion Dataset (San Vicente and Saralegi, 2016): Two small corpora in Basque containing news articles and reviews (music and movie domains). We merged them to create a larger dataset, containing a total of 224 reviews.

In addition, due to the lack of available sentenceor document-level corpora for Catalan or Galician, we opted for synthetic corpora:

- Synthetic Catalan SFU: An automatically translated version to $c a$ of the Spanish SFU, with $5 \%$ of the words from the original corpus considered as unknown by the translation tool.

- Synthetic Galician SFU: An automatically translated version to $g l$ of the Spanish SFU $(\approx$ $6.4 \%$ of the words not translated).

\subsection{Experiments}

We performed different experiments on binary polarity classification for knowing (a) the accuracy of the system, (b) the impact of the merged resources, and (c) the impact of the universal rules in monolingual and multilingual settings: 


\begin{tabular}{|l|rrrrr|}
\hline Lg & SL-O & SL+O & ML-O & ML+O & LKit \\
\hline \hline es & 60.00 & 75.75 & 63.75 & $\mathbf{7 6 . 5 0}$ & 58.75 \\
ca & 54.00 & 57.50 & 58.25 & $\mathbf{7 3 . 0 0}$ & - \\
gl & 60.75 & $\mathbf{7 3 . 0 0}$ & 60.00 & 70.00 & 50.25 \\
eu & 62.95 & 69.20 & 65.63 & $\mathbf{7 2 . 3 2}$ & - \\
pt & 60.50 & $\mathbf{6 7 . 3 5}$ & 57.29 & 65.01 & 60.55 \\
\hline
\end{tabular}

Table 4: Results of the different tests. In LKit we only evaluated the positive and negative results (it also classifies sentences with no polarity).

1. SL-O: Single lexica, no operations (baseline).

2. $M L-O$ : Multilingual lexica, no operations.

3. $S L+O$ : Single lexica with universal operations.

4. $M L+O$ : Multilingual lexica with universal operations.

The performance of our system was compared to LinguaKit (LKit), an open-source toolkit which performs supervised sentiment analysis in several languages (Gamallo et al., 2013; Gamallo and Garcia, 2017).

Table 4 shows the results of each of these models on the different corpora. The baseline $(S L$ $O$ ) obtained values between $54 \%(\mathrm{ca})$ and $62.95 \%$ $\mathrm{eu}$, results that are in line to those obtained by the supervised model. ${ }^{5}$ As we are not aware of available SA tools for $c a$, we could not compare our results with other systems. For Basque, San Vicente and Saralegi (2016) evaluated several lexica (both automatically translated and extracted, as well as with human annotation) in the same dataset used in this paper. They used a simple average polarity ratio classifier, which is similar to our baseline. Even if the lexica are different, their results are very similar to our $S L-O$ system ( $63 \%$ vs $62,95 \%$ ), and they also show that manually reviewing the lexica can boost the accuracy by up to $13 \%$.

The central columns of Table 4 show the results of using universal rules and a merged lexicon in the same datasets. In $g l$ and $p t$ the best values were obtained using individual lexica together with syntactic rules, while the Iberian system achieved the best results in the other languages.

Table 5 summarizes the impact that the rules have in both the monolingual and the multilingual setting, as well as the differences in performance due to the fusion process. Concerning the rules (columns 2 and 3), the results show that using the same set of universal rules improves the performance of the classifier in all the languages and settings. Their impact varies between 3.5 percentage

\footnotetext{
${ }^{5}$ LinguaKit was intended for tweets (not long texts).
}

\begin{tabular}{|l|rr|rr|}
\hline $\mathbf{L g}$ & $\mathbf{O}(\mathbf{S L})$ & $\mathbf{O}(\mathbf{M L})$ & $\mathbf{M L}(-\mathbf{O})$ & $\mathbf{M L}(+\mathbf{O})$ \\
\hline \hline es & 15.75 & 12.75 & 3.75 & 0.75 \\
ca & 3.50 & 14.75 & 4.25 & 15.5 \\
$\mathrm{gl}$ & 12.25 & 10.00 & -0.75 & -3.00 \\
eu & 6.25 & 6.69 & 2.68 & 3.12 \\
$\mathrm{pt}$ & 6.85 & 7.72 & -3.21 & -2.34 \\
\hline
\end{tabular}

Table 5: Impact of the operations $(\mathrm{O})$ with mono (SL) and multilingual lexica (ML) and of the ML with $(+\mathrm{O})$ and without operations (-O).

points ( $\mathrm{ca}$ ) and more than 15 (es) and, for each language, the rules provide a similar effect in monolingual and multilingual lexica (except for $c a$, with much higher values in the ML scenario).

The fusion of the different lexica had different results (columns 4 and 5 of Table 5): in $g l$ and $p t$, it had a negative impact (between $-0.75 \%$ and $-3.21 \%$ ) while in the other three the ML setting achieved better values (between 0.75 and 15.5 points, again with huge differences in $c a$ ). On average, using multilingual lexica had a positive impact of $1.3(-\mathrm{O})$ and 2.8 points $(+\mathrm{O})$. As mentioned, $c a$ has a different behaviour: the gain from rules when using monolingual lexica is about 3.50 points (lower than other languages), and the benefit of the ML lexicon without syntactic rules is of 4.25 points. However, when combining both the universal rules and the ML lexicon its performance increases $\approx 15$ points, turning out that the combination of these two factors is decisive.

In sum, the results of the experiments indicate that syntactic rules defined by means of a harmonized annotation can be used in several languages with positive results. Furthermore, the merging of monolingual lexica (some of them automatically translated) can be applied to perform multilingual SA with little impact in performance when compared to language-dependent systems.

\section{Conclusions and current work}

We built a single symbolic syntactic system for polarity classification that analyzes five official languages of the Iberian peninsula. With little effort we obtain robust results for many languages. As current work, we are working on texts harder to parse and low-resource languages: we developed a Galician corpus of manually labeled tweets, where SISA obtains between $62 \%$ and $65 \%$ accuracy for different settings, ${ }^{6}$ and plan to incorporate Kong et al. (2014) parser to improve its performance.

\footnotetext{
${ }^{6}$ This corpus is available at http: / / grupolys org / software/CHIOS-SISA/
} 


\section{References}

R. Agerri, J. Bermudez, and G. Rigau. 2014. IXA pipeline: Efficient and Ready to Use Multilingual NLP tools. In Proceedings of the 9th edition of the Language Resources and Evaluation Conference (LREC 2014), pages 3823-3828.

Waleed Ammar, George Mulcaire, Miguel Ballesteros, Chris Dyer, and Noah Smith. 2016. Many languages, one parser. Transactions of the Association for Computational Linguistics, 4:431-444.

P. P. Balage Filho, T. AS Pardo, and S. M. Aluísio. 2013. An evaluation of the Brazilian Portuguese LIWC dictionary for sentiment analysis. In Proceedings of the 9th Brazilian Symposium in Information and Human Language Technology (STIL), pages 215-219.

C. Bosco, M. Lai, V. Patti, F. M. Rangel Pardo, and P Rosso. 2016. Tweeting in the debate about catalan elections. In Proceedings of the Tenth International Conference on Language Resources and Evaluation (LREC 2016). Emotion and Sentiment Analysis Workshop., pages 67-70.

J. Brooke, M. Tofiloski, and M. Taboada. 2009. CrossLinguistic Sentiment Analysis: From English to Spanish. In Proceedings of RANLP 2009, Recent Advances in Natural Language Processing, pages 50-54, Bovorets, Bulgaria.

P. Carvalho, L. Sarmento, J. Teixeira, and M. J. Silva. 2011. Liars and saviors in a sentiment annotated corpus of comments to political debates. In Proceedings of the 49th Annual Meeting of the Association for Computational Linguistics: Human Language Technologies: short papers-Volume 2, pages 564-568. Association for Computational Linguistics.

H. Chen and H. Chen. 2016. Implicit Polarity and Implicit Aspect Recognition in Opinion Mining. In The 54th Annual Meeting of the Association for Computational Linguistics, pages 20-25.

F. L Cruz, J. A Troyano, B. Pontes, and F. J. Ortega. 2014. ML-SentiCon: Un lexicón multilingüe de polaridades semánticas a nivel de lemas. Procesamiento del Lenguaje Natural, 53:113-120.

M. L Forcada, M. Ginestí-Rosell, J. Nordfalk, J. O'Regan, S. Ortiz-Rojas, J. A. Pérez-Ortiz, F. Sánchez-Martínez, G. Ramírez-Sánchez, and F. M. Tyers. 2011. Apertium: a free/open-source platform for rule-based machine translation. $\mathrm{Ma}$ chine translation, 25(2):127-144.

P. Gamallo and M. Garcia. 2017. LinguaKit: uma ferramenta multilingue para a análise linguística e a extração de informação. Linguamática, 9(1):19-28.

P. Gamallo, M. García, and S. Fernández Lanza. 2013. TASS: A Naive-Bayes strategy for sentiment analysis on Spanish tweets. In XXIX Congreso de la
Sociedad Española de Procesamiento de Lenguaje Natural (SEPLN 2013). TASS 2013 - Workshop on Sentiment Analysis at SEPLN 2013, pages 126-132, Madrid, Spain.

M. Garcia and P. Gamallo. 2015. Yet Another Suite of Multilingual NLP Tools. In Languages, Applications and Technologies. Communications in Computer and Information Science, volume 563, pages 65-75. Springer.

L. F. Hurtado, F. Pla, and D. Buscaldi. 2015. ELiRFUPV en TASS 2015: Análisis de Sentimientos en Twitter. In Proceedings of TASS 2015: Workshop on Sentiment Analysis at SEPLN, pages 35-40.

L. Kong, N. Schneider, S. Swayamdipta, A. Bhatia, C. Dyer, and N. A. Smith. 2014. A Dependency Parser for Tweets. In Proceedings of the 2014 Conference on Empirical Methods in Natural Language Processing (EMNLP), pages 1001-1012, Doha, Qatar. ACL.

Eugenio Martínez-Cámara, M Teresa Martín-Valdivia, and L Alfonso Ureña-López. 2011. Opinion classification techniques applied to a spanish corpus. In International Conference on Application of Natural Language to Information Systems, pages 169-176. Springer.

R. T McDonald, J. Nivre, Y. Quirmbach-Brundage, Y. Goldberg, D. Das, K. Ganchev, K. B. Hall, S. Petrov, H. Zhang, O. Täckström, et al. 2013. Universal Dependency Annotation for Multilingual Parsing. In Proceedings of the 51st Annual Meeting of the Association for Computational Linguistics, pages 9297. Association for Computational Linguistics.

S. M Mohammad, S. Kiritchenko, and X. Zhu. 2013. NRC-Canada: Building the State-of-the-Art in Sentiment Analysis of Tweets. In Proceedings of the seventh international workshop on Semantic Evaluation Exercises (SemEval-2013), Atlanta, Georgia, USA.

J. Nivre, Ž. Agić, M. J. Aranzabe, M. Asahara, A. Atutxa, M. Ballesteros, J. Bauer, K. Bengoetxea, R. A. Bhat, C. Bosco, et al. 2015. Universal dependencies 1.2 .

L. Padró and E. Stanilovsky. 2012. Freeling 3.0: Towards wider multilinguality. In Proceedings of the 8th edition of the Language Resources and Evaluation Conference (LREC 2012), Istambul.

J. W. Pennebaker, M. E. Francis, and R. J. Booth. 2001. Linguistic inquiry and word count: LIWC 2001. Mahway: Lawrence Erlbaum Associates, page 71.

I. San Vicente and X. Saralegi. 2016. Polarity lexicon building: to what extent is the manual effort worth? In Proceedings of the Tenth International Conference on Language Resources and Evaluation (LREC 2016), Paris, France. European Language Resources Association (ELRA). 
X. Saralegi and I. San Vicente. 2013. Elhuyar at tass 2013. In Proceedings of the Workshop on Sentiment Analysis at SEPLN (TASS 2013), pages 143-150.

A. Shoukry and A. Rafea. 2012. Sentence-level Arabic sentiment analysis. In Collaboration Technologies and Systems (CTS), 2012 International Conference on, pages 546-550. IEEE.

M. J Silva, P. Carvalho, L. Sarmento, E. de Oliveira, and P. Magalhaes. 2009. The design of OPTIMISM, an opinion mining system for Portuguese politics. New trends in artificial intelligence: Proceedings of EPIA, pages 12-15.

R. Socher, A. Perelygin, J. Wu, J. Chuang, C. D Manning, A. Ng, and C. Potts. 2013. Recursive Deep Models for Semantic Compositionality Over a Sentiment Treebank. In EMNLP 2013. 2013 Conference on Empirical Methods in Natural Language Processing. Proceedings of the Conference, pages 1631-1642, Seattle, Washington, USA. ACL.

M. Souza and R. Vieira. 2012. Sentiment analysis on twitter data for portuguese language. In International Conference on Computational Processing of the Portuguese Language, pages 241-247. Springer.

M. Souza, R. Vieira, D. Busetti, R. Chishman, I. M. Alves, and Others. 2011. Construction of a portuguese opinion lexicon from multiple resources. In 8th Brazilian Symposium in Information and Human Language Technology, pages 59-66.

M. Taboada, J. Brooke, M. Tofiloski, K. Voll, and M. Stede. 2011. Lexicon-based methods for sentiment analysis. Computational Linguistics, 37(2):267-307.

M. Taulé, M. A. Martí, and M. Recasens. 2008. AnCora: Multilevel Annotated Corpora for Catalan and Spanish. In Proceedings of the Sixth International Conference on Language Resources and Evaluation (LREC'08), pages 96-101, Marrakech, Morocco.

K. Toutanova and C. D. Manning. 2000. Enriching the knowledge sources used in a maximum entropy partof-speech tagger. In Proceedings of the 2000 Joint SIGDAT conference on Empirical methods in natural language processing and very large corpora: held in conjunction with the 38th Annual Meeting of the Association for Computational LinguisticsVolume 13, pages 63-70.

P. D. Turney. 2002. Thumbs up or thumbs down?: semantic orientation applied to unsupervised classification of reviews. In Proceedings of the 40th Annual Meeting on Association for Computational Linguistics, ACL '02, pages 417-424, Stroudsburg, PA, USA. ACL.

D. Vilares, M. A. Alonso, and C. Gómez-Rodríguez. A syntactic approach for opinion mining on Spanish reviews. Natural Language Engineering, 21(01):139-163.
D. Vilares, M. A. Alonso, and C. Gómez-Rodríguez. 2015. On the usefulness of lexical and syntactic processing in polarity classification of Twitter messages. Journal of the Association for Information Science and Technology, 66(9):1799-1816.

D. Vilares, C. Gómez-Rodríguez, and M. A. Alonso. 2016. One model, two languages: training bilingual parsers with harmonized treebanks. In Proceedings of the 54th Annual Meeting of the Association for Computational Linguistics (Volume 2: Short Papers), pages 425-431, Berlin, Germany. Association for Computational Linguistics.

David Vilares, Carlos Gómez-Rodríguez, and Miguel A. Alonso. 2017. Universal, unsupervised (rule-based), uncovered sentiment analysis. Knowledge-Based Systems, 118:45-55.

J. Villena-Román, S. Lana-Serrano, E. MartínezCámara, and J C González C. 2013. TASS - Worshop on Sentiment Analysis at SEPLN. Procesamiento de Lenguaje Natural, 50:37-44.

D. T. Vo and Y. Zhang. 2016. Don't count, predict! an automatic approach to learning sentiment lexicons for short text. In Proceedings of the 54th Annual Meeting of the Association for Computational Linguistics (Volume 2: Short Papers), pages 219-224, Berlin, Germany. Association for Computational Linguistics. 